\title{
The angiotensin type 2 receptor weighs in on obesity: a promising therapeutic target?
}

\author{
Lucinda M Hilliard and Kate M Denton \\ Hypertension Research (2012) 35, 582-584; doi:10.1038/hr.2012.22; published online 16 February 2012
}

Excessive weight gain is one of the leading causes of increased arterial pressure in human essential hypertension. Given the rising obesity 'epidemic', there is an urgent need to further our understanding of the mechanisms responsible for weight-related changes in arterial pressure in order to improve treatment strategies. Extensive evidence exists that obesity-related hypertension is strongly associated with renal dysfunction. Like other forms of hypertension, the increased arterial pressure associated with obesity is accompanied by a shift in the renal pressure-natriuresis relationship, such that increased arterial pressure is required to maintain sodium homeostasis. ${ }^{1}$ This impairment in renal excretory function in obesity is initially thought to be primarily due to enhanced tubular reabsorption of sodium, since glomerular filtration rate and renal blood flow are actually increased. ${ }^{1}$

The renin-angiotensin system (RAS) has a pivotal role in the long-term regulation of arterial pressure by the kidney. Overactivity of the RAS is considered to have a major role in mediating obesity-related sodium retention. The principle effector peptide of the RAS, angiotensin II (AngII), elicits its effects via two main receptor types with equal affinity. ${ }^{2}$ The majority of the classically recognized actions of AngII are mediated by the angiotensin type 1 receptor $\left(\mathrm{AT}_{1} \mathrm{R}\right)$ including vasoconstriction, sodium reabsorption and vascular growth. In contrast, the angiotensin type 2 receptor $\left(\mathrm{AT}_{2} \mathrm{R}\right)$ directly opposes these pressor actions of AngII, by promoting vasodilation and natriuresis, as well as antiproli-

Professor KM Denton and Dr LM Hilliard are at the Department of Physiology, Monash University, Building 13F, Melbourne, Victoria 3800, Australia.

E-mail: kate.denton@monash.edu ferative and antigrowth effects. Additionally, other biologically active angiotensin metabolites have been identified in recent years, which can also directly oppose $\mathrm{AT}_{1} \mathrm{R}$ responses through their interaction with the $\mathrm{AT}_{2} \mathrm{R}$ and elicit natriuretic responses from the kidney, ${ }^{2}$ including angiotensin III (AngIII) and angiotensin (1-7) (Figure 1). In fact, accumulating evidence suggests that AngIII, rather than AngII, is actually the preferred ligand of the $\mathrm{AT}_{2} \mathrm{R}$ in the kidney. ${ }^{3}$

In obesity, multiple RAS components are reported to be significantly increased, including plasma renin activity, angiotensinogen, angiotensin-converting enzyme (ACE) and plasma AngII. ${ }^{1}$ In particular, increased AngII-induced renal $\mathrm{AT}_{1} \mathrm{R}$ activation is thought to be a key mechanism that contributes to the increased renal sodium retention and the resetting of pressure-natriuresis, leading to increased arterial pressure. Much emphasis has subsequently been placed on reducing the deleterious effects of the $A_{1} R$ to improve sodium excretion and reduce arterial pressure in obesity, through the use of angiotensin receptor blockers and ACE inhibitors. Numerous studies have shown that $\mathrm{AT}_{1} \mathrm{R}$ antagonism and ACE inhibition elicit natriuretic effects and arterial pressure reduction in obese humans and animal models, ${ }^{1}$ providing direct evidence that the antinatriuretic actions of endogenous AngII are exaggerated in obesity. However, more recently the role of the $\mathrm{AT}_{2} \mathrm{R}$ in obesityrelated hypertension has been attracting considerable attention, given its involvement in sodium homeostasis and arterial pressure regulation. Indeed, in obese rats, the enhanced natriuretic response to $\mathrm{AT}_{1} \mathrm{R}$ blockade was abolished with combined $\mathrm{AT}_{2} \mathrm{R}$ blockade, suggesting that unopposed $\mathrm{AT}_{2} \mathrm{R}$ stimulation contributes to the beneficial natriuretic effects of angiotensin receptor blockers. ${ }^{4}$ Moreover, experimental evidence from obese rats also suggests that the $\mathrm{AT}_{2} \mathrm{R}$ is upregulated in obesity. ${ }^{4}$ Collectively, these findings suggest that the $\mathrm{AT}_{2} \mathrm{R}$ may therefore be a promising innovative therapeutic target for improving renal function and reducing hypertension associated with obesity. In addition to counteracting the opposing effects of $\mathrm{AT}_{1} \mathrm{R}$ stimulation, $\mathrm{AT}_{2} \mathrm{R}$ stimulation may be particularly effective in obesity-related pathological states, where $\mathrm{AT}_{2} \mathrm{R}$ levels are increased.

The study of Ali and Hussain ${ }^{5}$ in an article appearing in this issue of Hypertension Research further investigates the functional role of the $\mathrm{AT}_{2} \mathrm{R}$ in obesity-related hypertension, using the obese Zucker rat (OZR) model. Previously, Hussain and colleagues identified a protective role for the $\mathrm{AT}_{2} \mathrm{R}$ against arterial pressure increases in OZR, demonstrating that chronic treatment of OZR for 2 weeks with the $\mathrm{AT}_{2} \mathrm{R}$ antagonist, PD123319, increased arterial pressure by $\sim 13 \mathrm{~mm} \mathrm{Hg}$, as compared with vehicle-treated control rats. ${ }^{6}$ To further substantiate these findings, in the current study, Ali and Hussain $^{5}$ examined the influence of direct $\mathrm{AT}_{2} \mathrm{R}$ stimulation on renal function in OZR using the highly specific $\mathrm{AT}_{2} \mathrm{R}$ agonist, Compound 21 (C21). Direct stimulation of the $\mathrm{AT}_{2} \mathrm{R}$ induced natriuretic effects in the OZR kidney. In anaesthetized OZR, in response to intravenous infusion of C21 ( 1 and $5 \mu \mathrm{g} \mathrm{kg}^{-1}$ per minute), urine flow and sodium excretion increased in a dose-dependent manner. Moreover, these effects were attenuated during combined C21 treatment with the $\mathrm{AT}_{2} \mathrm{R}$ antagonist, PD123319, supporting the role of the $\mathrm{AT}_{2} \mathrm{R}$ in these responses. We have also recently reported that the $A_{2} R$ has a significant role in the control of renal function. ${ }^{7,8}$ We showed that direct $\mathrm{AT}_{2} \mathrm{R}$ stimulation 


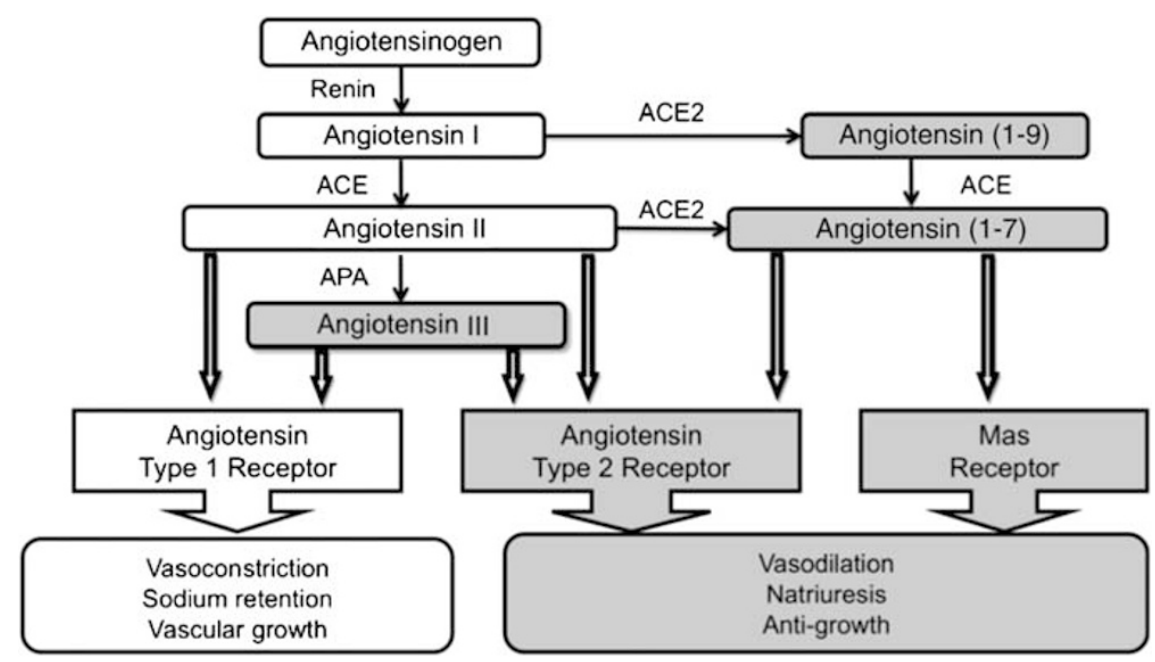

Figure 1 Schematic representation of the renin-angiotensin system (RAS), including the newly identified components of the depressor arm (highlighted). ACE, angiotensin-converting enzyme; ACE2, angiotensin-converting enzyme 2; APA, aminopeptidase A. A full color version of this figure is available at the Hypertension Research journal online.

using C21 has a pronounced influence on renal excretory and hemodynamic function, albeit in normotensive rats using a substantially lower dose $\left(0.028 \mu \mathrm{g} \mathrm{kg}^{-1}\right.$ per minute) ${ }^{8}$ Remarkably, in both studies, the renal response to $\mathrm{C} 21$ was observed without any inhibition of $\mathrm{AT}_{1} \mathrm{R}$ function, demonstrating the ability of $\mathrm{AT}_{2} \mathrm{R}$ agonism to directly modulate renal function.

In terms of the mechanisms that mediate $\mathrm{AT}_{2} \mathrm{R}$-mediated natriuresis, accumulating evidence suggests that stimulation of the nitric oxide/cGMP pathway may be involved. The natriuretic action of the $\mathrm{AT}_{2} \mathrm{R}$ has been linked to nitric oxide-mediated changes in the renal vasculature and tubules. It is well documented that $\mathrm{AT}_{2} \mathrm{R}$ activation in the vasculature stimulates the production of the bradykininnitric oxide-cGMP vasodilatory cascade. ${ }^{9}$ Moreover, this vasodilatory cascade has been associated with the inhibition of sodium transport in the renal tubules. ${ }^{9}$ In this context, a previous study by Hussain et al. ${ }^{10}$ suggests that $\mathrm{AT}_{2} \mathrm{R}$ activation via stimulation of the nitric oxide/cGMP pathway produces direct effects on sodium excretion in OZR by inhibiting proximal tubular $\mathrm{Na}^{+} \mathrm{K}^{+}$-ATPase activity, a response not observed in their lean rat model. In the current study, the $\mathrm{AT}_{2} \mathrm{R}$ mediated natriuresis observed was associated with increased urinary nitrate/nitrite and cGMP levels, further supporting this notion.

Despite the change in renal excretory function in response to C21 in OZR, the authors did not identify any significant change in glomerular filtration rate. However, fractional sodium excretion increased significantly, suggesting that changes in tubular sodium handling may be responsible for the C21-induced natriuresis. We also observed an increase in fractional sodium excretion in response to C21 administration in normotensive rats. ${ }^{8}$ The exact site of $\mathrm{AT}_{2} \mathrm{R}$ function that contributes to $\mathrm{AT}_{2} \mathrm{R}$-mediated natriuresis has not been completely elucidated. However, studies examining angiotensin receptor expression in the kidney have identified $\mathrm{AT}_{2} \mathrm{R}$ in the renal vasculature, as well as the renal tubules, particularly within proximal tubular cells, where the majority of sodium and water reabsorption occurs. ${ }^{9}$ To shed further light into this theory, in a separate set of experiments, Ali and Hussain ${ }^{5}$ measured lithium clearance as a marker of proximal tubular handling. Indeed, the fractional excretion of lithium was also significantly increased in response to C21 treatment. Moreover, C21induced natriuresis was observed during concomitant blockade of the major distal tubule sodium transporters and channels $(\mathrm{Na}-\mathrm{Cl}$ cotransporter and epithelial $\mathrm{Na}^{+}$channel), providing further support for proximal tubule involvement. However, as acknowledged by the authors, these results should be interpreted with caution and require further verification since there is considerable concern about the validity of lithium clearance as an index of proximal tubule sodium handling. Additionally, future examination of the contribution of the loop of Henle to $\mathrm{AT}_{2} \mathrm{R}$ mediated natriuresis is required.

In this study, as well as our previous study in normotensive rats ${ }^{8}$, acute C21 administration induced natriuresis and diuresis in the absence of any change in arterial pressure. In the current work, however, it is surprising that no change in arterial pressure was observed, considering this dose range ( $1 \mu \mathrm{g} \mathrm{kg}^{-1}$ per minute) of C21 was previously reported to elicit non-specific effects and led to increased arterial pressure in conscious spontaneously hypertensive rats during a 4-h infusion, indicating a lack of $\mathrm{AT}_{2} \mathrm{R}$ selectivity. ${ }^{11}$ The authors suggest this discrepancy may reflect the different experimental designs; the current study being performed in anaesthetized OZR in contrast to conscious spontaneously hypertensive rats. Alternatively, it may represent strain-dependent differences in sensitivity to C21 and/or differences in angiotensin receptor expression levels. Moreover, the influence of direct $\mathrm{AT}_{2} \mathrm{R}$ stimulation on renal hemodynamic function in obesity-related hypertension has not been thoroughly explored. As we reported previously, C21 administration induces significant vasodilatory effects in the renal vasculature. ${ }^{8}$ Since renal blood flow is reportedly already increased in obesity, at least during the early stages, whether direct $\mathrm{AT}_{2} \mathrm{R}$ stimulation would be entirely beneficial, or may in fact have detrimental effects, in this form of hypertension will depend on whether a deleterious increase in glomerular pressure results, and requires further interrogation.

It will also be important in future studies to examine the long-term antihypertensive and renoprotective effects of C21 in obesity. This is the next step required to establish whether $\mathrm{AT}_{2} \mathrm{R}$ stimulation could be considered as a novel antihypertensive therapy. Additionally, examination of the impact of $\mathrm{AT}_{2} \mathrm{R}$ stimulation during combined $\mathrm{AT}_{1} \mathrm{R}$ blockade would ascertain whether selective agonists for the $\mathrm{AT}_{2} \mathrm{R}$, like $\mathrm{C} 21$, could potentiate the long-term antihypertensive effects of existing therapies. Given that there are significant sex differences in the regulation of arterial pressure by the RAS, ${ }^{7,8}$ elucidation of the sex-specific renoprotective role 
of the $\mathrm{AT}_{2} \mathrm{R}$ in obesity-related hypertension is also required. Finally, with the functional role of the newly identified components of the depressor arm of the RAS in the regulation of arterial pressure still being explored (Figure 1), there remains much for us to learn, which may indeed reveal additional targets in the RAS suitable for therapeutic intervention. For instance, upstream RAS components required for AngIII production and degradation, could represent additional therapeutic targets for future investigations, given the major contribution of AngIII to $\mathrm{AT}_{2} \mathrm{R}$-mediated natriuresis. ${ }^{3}$

In conclusion, significant advances have recently been made in understanding the role of the $\mathrm{AT}_{2} \mathrm{R}$ in the regulation of arterial pressure and renal function. The findings of Ali and Hussain ${ }^{5}$ offer further insight into the mechanisms underlying renal dysfunction in obesity-related hypertension and the involve- ment of the $\mathrm{AT}_{2} \mathrm{R}$. It is now a matter for future investigations to establish whether selective $\mathrm{AT}_{2} \mathrm{R}$ agonists offer therapeutic potential in the setting of obesity.

\section{CONFLICT OF INTEREST}

The authors declare no conflict of interest.

1 Hall JE. The kidney, hypertension, and obesity. Hypertension 2003; 41: 625-633.

2 Jones ES, Vinh A, McCarthy CA, Gaspari TA, Widdop RE. AT2 receptors: functional relevance in cardiovascular disease. Pharmacol Ther 2008; 120: 292-316.

3 Padia SH, Kemp BA, Howell NL, Fournie-Zaluski MC, Roques BP, Carey RM. Conversion of renal angiotensin II to angiotensin III is critical for AT2 receptormediated natriuresis in rats. Hypertension 2008; 51 : 460-465.

4 Hakam AC, Hussain T. Renal angiotensin II type-2 receptors are upregulated and mediate the candesartan-induced natriuresis/diuresis in obese zucker rats. Hypertension 2005; 45: 270-275.
5 Ali $Q$, Hussain T. $A T_{2}$ receptor non-peptide agonist $\mathrm{C} 21$ promotes natriuresis in obese Zucker rats. Hypertens Res 2012; 35: 654-660.

6 Siddiqui $A H$, Ali $Q$, Hussain T. Protective role of angiotensin II subtype 2 receptor in blood pressure increase in obese zucker rats. Hypertension 2009; 53: 256-261.

7 Hilliard LM, Nematbakhsh M, Kett MM, Teichman E, Sampson AK, Widdop RE, Evans RG, Denton KM. Gender differences in pressure-natriuresis and renal autoregulation: role of the angiotensin type 2 receptor. Hypertension 2011; 57: 275-282.

8 Hilliard LM, Jones ES, Steckelings UM, Unger T, Widdop RE, Denton KM. Sex-specific influence of angiotensin type 2 receptor stimulation on renal function: A novel therapeutic target for hypertension. Hypertension 2011; 59: 409-414.

9 Siragy HM. The angiotensin II type 2 receptor and the kidney. J Renin Angiotensin Aldosterone Syst 2010; 11: 33-36.

10 Hakam AC, Hussain T. Angiotensin II type 2 receptor agonist directly inhibits proximal tubule sodium pump activity in obese but not in lean zucker rats. Hypertension 2006; 47: 1117-1124.

11 Bosnyak S, Welungoda IK, Hallberg A, Alterman M, Widdop RE, Jones ES. Stimulation of angiotensin AT2 receptors by the non-peptide agonist, compound 21 , evokes vasodepressor effects in conscious spontaneously hypertensive rats. Br J Pharmacol 2010; 159: 709-716. 